\title{
Approximations for Large Deflection of a Cantilever Beam under a Terminal Follower Force and Nonlinear Pendulum
}

\author{
H. Vázquez-Leal, ${ }^{1}$ Y. Khan, ${ }^{2}$ A. L. Herrera-May, ${ }^{3}$ U. Filobello-Nino, ${ }^{1}$ \\ A. Sarmiento-Reyes, ${ }^{4}$ V. M. Jiménez-Fernández, ${ }^{1}$ D. Pereyra-Díaz, ${ }^{1}$ A. Perez-Sesma, ${ }^{1}$ \\ R. Castaneda-Sheissa, ${ }^{1}$ A. Díaz-Sanchez, ${ }^{4}$ and J. Huerta-Chua ${ }^{5}$ \\ ${ }^{1}$ Electronic Instrumentation and Atmospheric Sciences School, University of Veracruz, Cto. Gonzalo Aguirre Beltrán S/N, \\ 91000 Xalapa, VER, Mexico \\ ${ }^{2}$ Department of Mathematics, Zhejiang University, Hangzhou 310027, China \\ ${ }^{3}$ Micro and Nanotechnology Research Center, University of Veracruz, Calzada Ruiz Cortines 455, 94292 Boca del Rio, VER, Mexico \\ ${ }^{4}$ National Institute for Astrophysics, Optics and Electronics, Luis Enrique Erro No. 1, Sta. María, 72840 Tonantzintla, PUE, Mexico \\ ${ }^{5}$ Facultad de Ingenieria Civil, Universidad Veracruzana, Venustiano Carranza S/N, Col. Revolucion, C.P. 93390, Poza Rica, \\ VER, Mexico
}

Correspondence should be addressed to H. Vázquez-Leal; hvazquez@uv.mx

Received 13 November 2012; Accepted 16 January 2013

Academic Editor: Gerhard-Wilhelm Weber

Copyright (C) 2013 H. Vázquez-Leal et al. This is an open access article distributed under the Creative Commons Attribution License, which permits unrestricted use, distribution, and reproduction in any medium, provided the original work is properly cited.

\begin{abstract}
In theoretical mechanics field, solution methods for nonlinear differential equations are very important because many problems are modelled using such equations. In particular, large deflection of a cantilever beam under a terminal follower force and nonlinear pendulum problem can be described by the same nonlinear differential equation. Therefore, in this work, we propose some approximate solutions for both problems using nonlinearities distribution homotopy perturbation method, homotopy perturbation method, and combinations with Laplace-Padé posttreatment. We will show the high accuracy of the proposed cantilever solutions, which are in good agreement with other reported solutions. Finally, for the pendulum case, the proposed approximation was useful to predict, accurately, the period for an angle up to $179.99999999^{\circ}$ yielding a relative error of 0.01222747 .
\end{abstract}

\section{Introduction}

Solving nonlinear differential equations is an important issue in science because many physical phenomena are modelled using such equations. During last century, the approximate solution of such equations was a task performed by hand using methods like the perturbation method. Nowadays, symbolic software like Maple or Mathematica allow researchers to calculate highly accurate approximate solutions using new methods like the homotopy perturbation method (HPM) [1-38]. The HPM method is one of the most famous analytic techniques for nonlinear differential equations, which is widely applied in science and engineering.

In $[28,39]$ was reported the nonlinearities distribution homotopy perturbation method (NDHPM) as an extended version of HPM that allows the distribution of the nonlinearities between the different iterations of HPM method. The main advantage of this process is the increase in the number of possible iterations by constructing easier to solve linear equations for HPM procedure. Additionally, several works reported that a Laplace-Padé posttreatment [40-49] of power series solutions obtained by HPM can improve accuracy and convergence to the exact solution. Therefore, in this work, we propose the use of the aforementioned methods to obtain approximate solutions for two nonlinear problems, related by to same nonlinear differential equation. The first one is the large deflection of a cantilever beam under a terminal follower force [50-55]. This kind of structures arises in marine riser and other practical applications of civil engineering. The mathematical formulation of such a problem yields a nonlinear two-point boundary-value problem, which usually can be solved by numerical methods [50]. The second one is the nonlinear pendulum problem [56-58], which is modelled 
by the same equation of the Cantilever's case study. The pendulum or systems containing the pendulums are wellstudied complex phenomen [59]. For both problems, the results exhibited high accuracy using our proposed solutions, which are in good agreement to the results reported by other works. In particular, for the pendulum case, we succeeded to predict, accurately, the period until an initial angle up to $179.99999999^{\circ}$ achieving a relative error of 0.01222747 .

This paper is organized as follows. In Section 2, the basic idea of the HPM method is provided. We give an introduction to NDHPM method in Section 3. In Section 4, the basic concept of Padé approximants is explained. The Laplace-Padé coupling with NDHPM and HPM methods is presented in Section 5. In Sections 6 and 7, the cantilever and pendulum problems are solved, respectively. In addition, a discussion on the results is presented in Section 8. Finally, a brief conclusion is given in Section 9.

\section{Basic Concept of HPM}

It can be considered that a nonlinear differential equation can be expressed as

$$
A(u)-f(r)=0, \quad \text { where } r \in \Omega,
$$

having boundary condition as

$$
B\left(u, \frac{\partial u}{\partial \eta}\right)=0, \quad \text { where } r \in \Gamma,
$$

where $A$ is a general differential operator, $f(r)$ is a known analytic function, $B$ is a boundary operator, $\Gamma$ is the boundary of domain $\Omega$, and $\partial u / \partial \eta$ denotes differentiation along the normal drawn outwards from $\Omega$ [50]. The $A$ operator, generally, can be divided into two operators, $L$ and $N$, which are linear and nonlinear operators, respectively. Hence, (1) can be rewritten as

$$
L(u)+N(u)-f(r)=0 .
$$

Now, a possible homotopy formulation is

$$
\begin{aligned}
H(v, p)= & (1-p)\left[L(v)-L\left(u_{0}\right)\right] \\
& +p(L(v)+N(v)-f(r))=0, \quad p \in[0,1],
\end{aligned}
$$

where $u_{0}$ is the initial approximation for (3) which satisfies the boundary conditions and $p$ is known as the perturbation homotopy parameter.

For the HPM method [4-7], we assume that the solution for (4) can be written as a power series of $p$

$$
v=p^{0} v_{0}+p^{1} v_{1}+p^{2} v_{2}+\cdots
$$

Considering that $p \rightarrow 1$, results that the approximate solution of (1) is

$$
u=\lim _{p \rightarrow 1} v=v_{0}+v_{1}+v_{2}+\cdots .
$$

The series (6) is convergent on most cases as reported in $[4,7,20,21]$.

\section{HPM Method with Nonlinearities Distribution}

Recent reports [28, 39] have introduced the NDHPM method, which eases the searching process of solutions for (3) and reduces the complexity of solving differential equations. As first step, a modified homotopy is introduced:

$$
\begin{aligned}
H(v, p)= & (1-p)\left[L(v)-L\left(u_{0}\right)\right] \\
& +p(L(v)+N(v, p)-f(r, p))=0, \\
& p \in[0,1] .
\end{aligned}
$$

It can be noticed that the homotopy function (7) is essentially the same as (4), except for the nonlinear operator $N$ and the nonhomogeneous function $f$, which embeds the homotopy parameter $p$. The arbitrary introduction of $p$ within the differential equation is a strategy to redistribute the nonlinearities between the successive iterations of the HPM method and, thus, increase the probabilities of finding the sought solution.

Again, we establish that

$$
v=\sum_{i=0}^{\infty} v_{i} p^{i}
$$

Considering that $p \rightarrow 1$ turns out that the approximate solution for (1) is

$$
u=\lim _{p \rightarrow 1} v=\sum_{i=0}^{\infty} v_{i}
$$
[28].

The convergence of the NDHPM method is exposed in

\section{Padé Approximants}

A rational approximation to $f(x)$ on $[a, b]$ is the quotient of two polynomials $P_{N}(x)$ and $Q_{M}(x)$ of degrees $N$ and $M$, respectively. We use the notation $R_{N, M}(x)$ to denote this quotient. The $R_{N, M}(x)$ Padé approximations to a function $f(x)$ are given by $[49,60]$

$$
R_{N, M}=\frac{P_{N}(x)}{Q_{M}(x)}, \quad \text { for } a \leq x \leq b .
$$

The method of Padé requires that $f(x)$ and its derivative should be continuous at $x=0$. The polynomials used in (10) are

$$
\begin{aligned}
& P_{N}(x)=p_{0}+p_{1} x+p_{2} x^{2}+\cdots+p_{N}(x), \\
& Q_{M}(x)=q_{0}+q_{1} x+q_{2} x^{2}+\cdots+q_{M}(x) .
\end{aligned}
$$

The polynomials in (11) are constructed so that $f(x)$ and $R_{N, M}(x)$ agree at $x=0$ and their derivatives up to $N+M$ agree at $x=0$. For the case where $Q_{0}(x)=1$, the approximation is just the Maclaurin expansion for $f(x)$. For a fixed value of $N+M$ the error is the smallest when $P_{N}(x)$ and $Q_{M}(x)$ 
have the same degree or when $P_{N}(x)$ has a degree higher than $Q_{M}(x)$.

Notice that the constant coefficient of $Q_{M}$ is $q_{0}-1$. This is permissible because it can be noted that 0 and $R_{N, M}(x)$ are not changed when both $P_{N}(x)$ and $Q_{M}(x)$ are divided by the same constant. Hence, the rational function $R_{N, M}(x)$ has $N+M+1$ unknown coefficients. Assume that $f(x)$ is analytic and has the Maclaurin expansion

$$
f(x)=a_{0}+a_{1} x+a_{2} x^{2}+\cdots+a_{k} x^{k}+\cdots .
$$

And, from the difference $f(x) Q_{M}(x)-P_{N}(x)=Z(x)$

$$
\left\lceil\sum_{i=0}^{\infty} a_{i} x^{i}\right\rceil\left\lceil\sum_{i=0}^{M} q_{i} x^{i}\right\rceil-\left\lceil\sum_{i=0}^{N} p_{i} x^{i}\right\rceil=\left\lceil\sum_{i=N+M+1}^{\infty} c_{i} x^{i}\right\rceil .
$$

The lower index $j=N+M+1$ in the summation at the right side of (13) is chosen because the first $N+M$ derivatives of $f(X)$ and $R_{N, M}(x)$ should agree at $x=0$.

When the left side of (13) is multiplied out and coefficients of the powers of $x^{i}$ are set equal to zero for $k=0,1,2, \ldots, N+$ $M$, this results in a system of $N+M+1$ linear equations:

$$
\begin{gathered}
a_{0}-p_{0}=0 \\
q_{1} a_{0}+a_{1}-p_{1}=0 \\
q_{2} a_{0}+q_{1} a_{1}+a_{2}-p_{2}=0 \\
q_{3} a_{0}+q_{2} a_{1}+q_{1} a_{2}+a_{3}-p_{3}=0 \\
q_{M} a_{N-M}\left|q_{M-1} a_{N-M-1}\right| a_{N}-p_{N}=0, \\
q_{M} a_{N-M+1}+q_{M-1} a_{N-M+2}+\cdots+q_{1} a_{N}+a_{N+2}=0 \\
q_{M} a_{N-M+2}+q_{M-1} a_{N-M+3}+\cdots+q_{1} a_{N+1}+a_{N+3}=0 \\
\vdots \\
q_{M} a_{N}+q_{M} a_{N+1}+\cdots+q_{1} a_{N+M+1}+a_{N+M}=0 .
\end{gathered}
$$

Notice that in each equation the sum of the subscripts on the factors of each product is the same, and this sum increases consecutively from 0 to $N+M$. The $M$ equations in (15) involve only the unknowns $q_{1}, q_{2}, \ldots, q_{M}$ and must be solved first. Then, the equations in (14) are successively used to find $p_{1}, p_{2}, \ldots, p_{N}[49]$

\section{Laplace-Padé Posttreatment of Power Series Solutions}

The coupling of Laplace transform and Padé approximant [40] is used to deal with the lost information of truncated power series [41-49]. The process can be summarized as follows.

(1) First, Laplace transformation is applied to a power series solution obtained by HPM or NDHPM methods.

(2) Next, $1 / t$ is written in place of $s$.

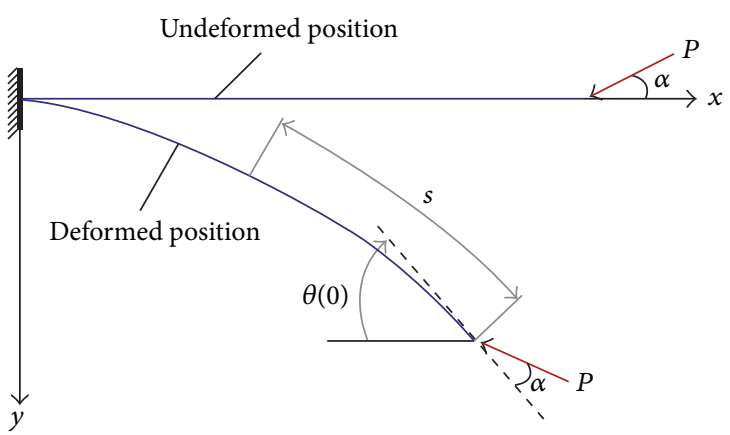

Figure 1: A cantilever beam under terminal follower force.

(3) Afterwards, we convert the transformed series into a meromorphic function by forming its Padé approximant of order $[N / M] . N$ and $M$ are arbitrarily choses, but they should be smaller than the power series order. In this step, the Padé approximant extends the domain of the truncated series solution to obtain better accuracy and convergence.

(4) Then, $1 / s$ is written in place of $t$.

(5) Finally, by using the inverse Laplace $s$ transformation, we obtain the modified approximate solution.

The Laplace-Padé posttreatment of HPM and NDHPM methods will be referred throughout the rest of this paper as LPHPM and LPNDHPM, respectively.

\section{Solution of Cantilever Problem}

Beams subjected to follower load arise in many problems of structural engineering like lumbar spine in biomechanics, smart structures applications, and loads applied to structural components by cables in tension, among others. The governing equations of such structures involve the effects of nonlinearities due to large deformations and material properties [50-55].

In this work, we study the large-deflection problem of a cantilever beam under a tip-concentrated follower force as depicted in Figure 1. The angle $\alpha$ of inclination of the force with respect to the deformed axis of the beam remains unchanged during deformation. We assume that the center of the Cartesian system $(x, y)$ is located at the fixed end and $\theta$ is the local angle of inclination of the centroidal axis beam. Therefore, the mathematical formulation of this problem yields the following nonlinear two-point boundaryvalue problem:

$$
\theta^{\prime \prime}+\lambda \sin [\theta+\alpha-\theta(0)]=0, \quad \theta^{\prime}(0)=0, \quad \theta(1)=0,
$$

where prime denotes differentiation with respect to $\eta$. Additionally, the related dimensionless quantities are defined as

$$
\eta=\frac{s}{l}, \quad \xi=\frac{x}{l}, \quad \zeta=\frac{y}{l}, \quad \lambda=\frac{l^{2} P}{E l},
$$

where $l$ represents the length of the originally straight elastic cantilever beam, $E l$ is the flexural rigidity, $s$ is the arc length 
measured from the tip, and $P$ is the terminal follower force with constant angle $\alpha$.

In [50] is reported that (16) can be reduced to an initialvalue problem by the following transformation:

$$
\phi(\eta)=\theta(\eta)+\alpha-\theta(0)
$$

resulting in the following initial value problem:

$$
\phi^{\prime \prime}+\lambda \sin (\phi)=0, \quad \phi(0)=\alpha, \quad \phi^{\prime}(0)=0,
$$

with the extra condition:

$$
\phi(1)-\alpha+\theta(0)=0
$$

where the slope of $\theta(\eta)$ can be obtained by using (18) and (20), resulting in the following:

$$
\theta(\eta)=\phi(\eta)-\phi(1)
$$

Finally, the deformed curve of beam axis can be calculated from solution of the slope $\theta(\eta)$ as

$$
\begin{aligned}
& \xi(\eta)=\int_{\eta}^{1} \cos (\theta) d \tilde{\eta} \\
& \zeta(\eta)=\int_{\eta}^{1} \sin (\theta) d \widetilde{\eta} .
\end{aligned}
$$

6.1. Solution Obtained by Using NDHPM Method. In order to circumvent difficulties due to the nonlinear sin term of (19), we propose to use a seventh-order Taylor series expansion, resulting in the following:

$$
\begin{gathered}
\phi^{\prime \prime}+\lambda\left(\phi-\frac{1}{6} \phi^{3}+\frac{1}{120} \phi^{5}-\frac{1}{5040} \phi^{7}\right)=0, \\
\phi(0)=\alpha, \quad \phi^{\prime}(0)=0,
\end{gathered}
$$

and by using (7) and (23), we establish the following homotopy equation:

$$
\begin{aligned}
& (1-p)\left(L(v)-L\left(\phi_{0}\right)\right) \\
& \quad+p\left(v^{\prime \prime}+\lambda\left(v-\frac{1}{6} v^{3}+\frac{1}{120} v^{5} p-\frac{1}{5040} v^{7} p^{2}\right)\right)=0
\end{aligned}
$$

where homotopy parameter $p$ has been arbitrarily embedded into the power series of Taylor's expansion, and linear operator $L$ is

$$
v^{\prime \prime}+\lambda v
$$

and trial function $\phi_{0}$ is

$$
\phi_{0}=\alpha \cos (\lambda \eta)
$$

Substituting (8) into (24) and reordering and equating terms with the same $p$ powers, we obtain the following linear set of differential equations:

$$
\begin{gathered}
p^{0}: v_{0}^{\prime \prime}+\lambda v_{0}=0, \quad v_{0}(0)=\alpha, \quad v_{0}^{\prime}(0)=0, \\
p^{1}: v_{1}^{\prime \prime}+\lambda v_{1}-\frac{1}{6} \lambda v_{0}^{3}=0, \\
v_{1}(0)=0, \quad v_{1}^{\prime}(0)=0, \\
p^{2}: v_{2}^{\prime \prime}+\lambda v_{2}+\frac{1}{120} \lambda v_{0}^{5}-\frac{1}{2} \lambda v_{0}^{2} v_{1}=0, \\
v_{2}(0)=0, \quad v_{2}^{\prime}(0)=0, \\
p^{3}: v_{3}^{\prime \prime}+\lambda v_{3}-\frac{1}{2} \lambda v_{0} v_{1}^{2}-\frac{1}{5040} \lambda v_{0}^{7} \\
+\frac{1}{24} \lambda v_{0}^{4} v_{1}-\frac{1}{2} \lambda v_{0}^{2} v_{2}=0, \\
v_{3}(0)=0, \quad v_{3}^{\prime}(0)=0 .
\end{gathered}
$$

By solving (27), we obtain

$$
\begin{aligned}
& v_{0}=\alpha \cos (\widehat{\lambda} \eta) \\
& v_{1}=-\frac{1}{48} \alpha^{3}\left(-\cos (\hat{\lambda} \eta)+\cos ^{3}(\widehat{\lambda} \eta)-3 \sin (\widehat{\lambda} \eta) \hat{\lambda} \eta\right) \text {, } \\
& v_{2}=\frac{1}{15360} \alpha^{5}(23 \cos (\hat{\lambda} \eta) \\
& -60 \cos ^{2}(\hat{\lambda} \eta) \sin (\hat{\lambda} \eta) \hat{\lambda} \eta \\
& +15 \sin (\hat{\lambda} \eta) \hat{\lambda} \eta-35 \cos ^{3}(\hat{\lambda} \eta) \\
& \left.+12 \cos ^{5}(\hat{\lambda} \eta)-30 \cos (\hat{\lambda} \eta) \hat{\lambda}^{2} \eta^{2}\right) \text {, } \\
& v_{3}=-\frac{1}{5160960} \alpha^{7}\left(-593 \cos \left(\widehat{\lambda}_{\eta}\right)\right. \\
& +1148 \cos ^{3}\left(\hat{\lambda}_{\eta}\right)-609 \sin (\hat{\lambda} \eta) \hat{\lambda} \eta \\
& -735 \cos ^{5}(\hat{\lambda} \eta)+1365 \cos (\hat{\lambda} \eta) \hat{\lambda}^{2} \eta^{2} \\
& +180 \cos ^{7}(\hat{\lambda} \eta)-1260 \sin \left(\widehat{\lambda}_{\eta}\right) \\
& \times \hat{\lambda} \eta \cos ^{7}(\hat{\lambda} \eta) x+2100 \cos ^{2}(\hat{\lambda} \eta) \\
& \times \sin (\hat{\lambda} \eta) \hat{\lambda} \eta+210 \sin (\hat{\lambda} \eta) \hat{\lambda}^{3} \eta^{3} \\
& \left.-1890 \widehat{\lambda}^{2} \cos ^{3}(\widehat{\lambda} \eta) \eta^{2}\right),
\end{aligned}
$$

where $\widehat{\lambda}=\sqrt{\lambda}$.

Substituting solutions (28) into (8) and calculating the limit when $p \rightarrow 1$ results in the third-order approximation:

$$
\phi(\eta)=\lim _{p \rightarrow 1}\left(\sum_{i=0}^{3} v_{i} p^{i}\right)=v_{0}+v_{1}+v_{2}+v_{3} .
$$


6.2. Solution Calculated by Using HPM and LPHPM Methods. By using (4) and (23), we establish the following homotopy equation:

$$
\begin{aligned}
& (1-p)\left(L(v)-L\left(\phi_{0}\right)\right) \\
& \quad+p\left(v^{\prime \prime}+\lambda\left(v-\frac{1}{6} v^{3}+\frac{1}{120} v^{5}-\frac{1}{5040} v^{7}\right)\right)=0
\end{aligned}
$$

where linear operator $L$ is

$$
v^{\prime \prime}
$$

and trial function $\phi_{0}$ is

$$
\phi_{0}=\alpha
$$

Substituting (5) into (30) and reordering and equating terms with the same $p$ powers, we obtain the following set of linear differential equations:

$$
\begin{gathered}
p^{0}: v_{0}^{\prime \prime}=0, \quad v_{0}(0)=\alpha, \quad v_{0}^{\prime}(0)=0 \\
p^{1}: v_{1}^{\prime \prime}-\frac{1}{5040} \lambda v_{0}^{7}-\frac{1}{6} \lambda v_{0}^{3}+\lambda v_{0}+\frac{1}{120} \lambda v_{0}^{5}=0, \\
v_{1}(0)=0, \quad v_{1}^{\prime}(0)=0 \\
p^{2}: v_{2}^{\prime \prime}+\frac{1}{24} \lambda v_{0}^{4} v_{1}+\lambda v_{1}-\frac{1}{2} \lambda v_{0}^{2} v_{1}-\frac{1}{720} \lambda v_{0}^{6} v_{1}=0, \\
v_{2}(0)=0, \quad v_{2}^{\prime}(0)=0
\end{gathered}
$$

By solving (33), we obtain

$$
\begin{gathered}
v_{0}=\alpha \\
v_{1}=\left(\frac{1}{10080} \lambda \alpha^{7}+\frac{1}{12} \lambda \alpha^{3}-\frac{1}{2} \lambda \alpha-\frac{1}{240} \lambda \alpha^{5}\right) \eta^{2} \\
v_{2}=\left(-\frac{1}{1209600} \lambda^{2} \alpha^{11}-\frac{1}{1890} \lambda^{2} \alpha^{7}+\frac{1}{180} \lambda^{2} \alpha^{5}\right. \\
+\frac{41}{1451520} \lambda^{2} \alpha^{9}-\frac{1}{36} \lambda^{2} \alpha^{3} \\
\left.+\frac{1}{24} \lambda^{2} \alpha+\frac{1}{87091200} \lambda^{2} \alpha^{13}\right) \eta^{4}
\end{gathered}
$$

where the subsequent iterations are calculated using Maple CAS software.

Substituting solutions (34) into (5) and calculating the limit when $p \rightarrow 1$, we can obtain the 18th-order approximation:

$$
\phi(\eta)=\lim _{p \rightarrow 1}\left(\sum_{i=0}^{18} v_{i} p^{i}\right) .
$$

Expression (35) is too long to be written here; nevertheless, it can be simplified if we consider a particular case. For instance, when $\alpha=\pi / 2$, it results in the following:

$$
\begin{aligned}
u(\eta)= & -5.0091602 \times 10^{-5} \eta^{10} \lambda^{5}-3.7265881 \times 10^{-5} \eta^{4} \lambda^{2} \\
& +0.004184205840 \eta^{6} \lambda^{3} \\
& +7.200012957 \times 10^{-17} \eta^{36} \lambda^{18}-0.4999215505 \eta^{2} \lambda \\
& +3.48692001 \times 10^{-9} \eta^{16} \lambda^{8} \\
& -4.36006 \times 10^{-12} \eta^{26} \lambda^{13}-1.4 \times 10^{-15} \eta^{34} \lambda^{17} \\
& -3.618364186 \times 10^{-7} \eta^{12} \lambda^{6} \\
& +7.89 \times 10^{-14} \eta^{30} \lambda^{15}-2.448310334 \times 10^{-12} \eta^{24} \lambda^{12} \\
& +2.455105652 \times 10^{-10} \eta^{22} \lambda^{11} \\
& +0.0000008161908993 \eta^{14} \lambda^{7}-1.686 \times 10^{-15} x^{32} \lambda^{16} \\
& -1.409132166 \times 10^{-8} \eta^{18} \lambda^{9} \\
& +4.567238741 \times 10^{-11} \eta^{20} \lambda^{10}+7.07 \times 10^{-14} \eta^{28} \lambda^{14} \\
& +1.570796327-7.024412 \times 10^{-6} \eta^{8} \lambda^{4} .
\end{aligned}
$$

By using (36) and (21) we can calculate, approximately, the deflection angle $\theta(0)$; nevertheless, accuracy can be increased applying the Laplace-Padé posttreatment. First, Laplace transformation is applied to (36); then, $1 / \eta$ is written in place of $s$. Afterwards, Padé approximant [8/8] is applied and $1 / s$ is written in place of $\eta$. Finally, by using the inverse Laplace $s$ transformation, we obtain the modified approximate solution. For instance, for $\lambda=13.75$, the result of LPHPM is

$$
\begin{aligned}
u(\eta)= & -0.1295812455566806 \\
& \times 10^{-3} \exp (-5.536309139671352 \eta) \\
& \times \cos (14.54887311024889 \eta) \\
& +0.3532982009243434 \\
& \times 10^{-4} \exp (-5.536309139671352 \eta) \\
& \times \sin (14.54887311024889 \eta) \\
& -0.2686099209980136 \\
& \times 10^{-1} \cos (8.783120641372600 \eta) \\
& +1.597916481385812 \\
& \times \cos (3.150018868129787 \eta) \\
& -0.1295812455566806 \\
& \times 10^{-3} \exp (5.536309139671352 \eta) \\
& \times \cos (14.54887311024889 \eta) \\
& -0.3532982009243434 \\
& \times 10^{-4} \exp (5.536309139671352 \eta) \\
& \times \sin (14.54887311024889 \eta)
\end{aligned}
$$


On one side, for $\lambda=13.75$, the exact result is $\theta(0)=180^{\circ}$ [51]. On the other side, by using (21), we can calculate the deflection angle using (36) and (37) approximations, resulting in $240146^{\circ}$ and $179.1119^{\circ}$, respectively. Therefore, for this case, the Laplace-Padé posttreatment was relevant in order to deal with the HPM truncated power series, resulting a notorious increase of accuracy (see Table 1).

6.3. Power Series Solution by Using NDHPM and LaplacePadé Posttreatment. By using (7) and (23), we establish the following homotopy equation:

$$
\begin{aligned}
& (1-p)\left(L(v)-L\left(u_{0}\right)\right) \\
& \quad+p\left(v^{\prime \prime}+\lambda\left(v-\frac{1}{6} v^{3} p+\frac{1}{120} v^{5} p^{2}-\frac{1}{5040} v^{7} p^{3}\right)\right)=0
\end{aligned}
$$

where homotopy parameter $p$ has been arbitrarily embedded into the power series of Taylor expansion, linear operator $L$, and trial function $\phi_{0}$ are taken from (31) and (32), respectively.

Substituting (8) into (38), reordering and equating terms with the same $p$ powers, we obtain the following set of linear differential equations:

$$
\begin{gathered}
p^{0}: v_{0}^{\prime \prime}=0, \quad v_{0}(0)=\alpha, \quad v_{0}^{\prime}(0)=0, \\
p^{1}: v_{1}^{\prime \prime}+\lambda v_{0}=0, \quad v_{1}(0)=0, \quad v_{1}^{\prime}(0)=0, \\
p^{2}: v_{2}^{\prime \prime}-\frac{1}{6} \lambda v_{0}^{3}+\lambda v_{1}=0, \quad v_{2}(0)=0, \quad v_{2}^{\prime}(0)=0 .
\end{gathered}
$$

Solving (39), we obtain

$$
\begin{gathered}
v_{0}=\alpha, \\
v_{1}=-\frac{1}{2} \lambda \alpha \eta^{2}, \\
v_{2}=\frac{1}{24} \lambda^{2} \alpha \eta^{4}+\frac{1}{12} \lambda \alpha^{3} \eta^{2},
\end{gathered}
$$

where the subsequent iterations are calculated using Maple CAS software.

Substituting solutions (40) into (8) and calculating the limit when $p \rightarrow 1$, we can obtain the 18th-order approximation:

$$
\phi(\eta)=\lim _{p \rightarrow 1}\left(\sum_{i=0}^{18} v_{i} p^{i}\right) .
$$

Expression (41) is too long to be written here; nevertheless, it can be simplified considering a particular case. For instance, a terminal follower normal angle $\alpha=\pi / 2$, resulting

$$
\begin{aligned}
\phi(\eta)= & 4.222646520 \times 10^{-42} \lambda^{18} \eta^{36}+1.140293002 \\
& \times 10^{-24} \lambda^{17} \eta^{34}+4.131839790 \times 10^{-17} \lambda^{16} \eta^{32} \\
& +1.098177235 \times 10^{-12} \lambda^{15} \eta^{30}+7.059044113 \\
& \times 10^{-10} \lambda^{14} \eta^{28}+5.042078503 \times 10^{-8} \lambda^{13} \eta^{26} \\
& +8.416343250 \times 10^{-7} \lambda^{12} \eta^{24}+4.617471554 \\
& \times 10^{-6} \lambda^{11} \eta^{22}+9.600210952 \times 10^{-6} \lambda^{10} \eta^{20} \\
& +7.77303550 \times 10^{-6} \lambda^{9} \eta^{18}+2.32539526 \\
& \times 10^{-6} \lambda^{8} \eta^{16}+1.0311191 \times 10^{-6} \lambda^{7} \eta^{14} \\
& -3.576556 \times 10^{-7} \lambda^{6} \eta^{12}-5.00915992 \\
& \times 10^{-5} \lambda^{5} \eta^{10}-7.024403934 \times 10^{-6} \eta^{8} \lambda^{4} \\
& +4.184205821 \times 10^{-3} \eta^{6} \lambda^{3}-3.726587 \\
& \times 10^{-5} \lambda^{2} \eta^{4}-0.4999215505 \lambda \eta^{2}+1.570796327
\end{aligned}
$$

In order to increase accuracy of $\theta(0),(42)$ is transformed by the Padé approximation and Laplace transformation. First, Laplace transformation is applied to (42) and then $1 / \eta$ is written in place of $s$. Then, Padé approximant [8/8] is applied and $1 / s$ is written in place of $\eta$. Finally, by using the inverse Laplace $s$ transformation, we obtain the modified approximate solution. For example, given $\lambda=13.75$, the resultant LPNDHPM approximation is

$$
\begin{aligned}
u(\eta)= & -0.1295804634 \times 10^{-3} \exp (-5.536309145 \eta) \\
& \times \cos (14.54887309 \eta) \\
& +0.3532927940 \times 10^{-4} \exp (-5.536309145 \eta) \\
& \times \sin (14.54887309 \eta) \\
& -0.2686099338 \times 10^{-1} \cos (8.783120717 \eta) \\
& +1.597916481 \cos (3.150018864 \eta) \\
& -0.1295804634 \times 10^{-3} \exp (5.536309145 \eta) \\
& \times \cos (14.54887309 \eta) \\
& -0.3532927940 \times 10^{-4} \exp (5.536309145 \eta) \\
& \times \sin (14.54887309 \eta) .
\end{aligned}
$$

Now, by using (21), we can calculate $\theta(0)$ for (42) and (43) approximations. Results for NDHPM and LPNDHPM are $-5.39274 e(+9)^{\circ}$ and $180.0345^{\circ}$, respectively (see Table 1 ). In fact, for $\lambda=13.75$, the exact result is $\theta(0)=180^{\circ}$; therefore, the Laplace-Padé after-treatment was a key factor to increase accuracy of NDHPM truncated power series. 
TABLE 1: Terminal slope $\theta(0)$ of cantilever beam under normal follower forces $(\alpha=\pi / 2)$.

\begin{tabular}{lcccccccc}
\hline$\lambda$ & $\theta(0)^{\circ}[51]$ & S.M. [53] & NDHPM (29) & HPM (35) & LPHPM (37) & NDHPM (41) & LPNDHPM (43) & HPM [50] \\
\hline 0.7010 & 20 & 19.50 & 20.0016 & 19.9981 & 19.9981 & 19.998 & 19.9981 & 19.9981 \\
1.4195 & 40 & 39.03 & 39.9784 & 39.9957 & 39.9958 & 39.9487 & 39.9957 & 39.9976 \\
2.1755 & 60 & 58.61 & 59.9681 & 59.9964 & 59.9975 & 56.1523 & 59.9959 & 60.0066 \\
2.9941 & 80 & 78.25 & 79.9905 & 79.9947 & 80.0057 & -36.9334 & 79.9934 & 80.0233 \\
3.9117 & 100 & 98.00 & 100.0498 & 99.9943 & 100.0610 & -2104.49 & 99.9999 & 100.0504 \\
4.9872 & 120 & 117.88 & 120.1375 & 120.001 & 120.2963 & -33644.8 & 120.0574 \\
6.3339 & 140 & 137.93 & 140.2400 & 140.57 & 141.1245 & -523001 & 140.3214 \\
8.2421 & 160 & 158.27 & 160.2464 & 221.529 & 163.5890 & $-1.13840 e+07$ & 161.2001 & 140.1092 \\
13.7500 & 180 & 179.97 & 178.7521 & 240146 & 179.1119 & $-5.39274 e+09$ & 180.0345 & 179.1172 \\
\hline
\end{tabular}

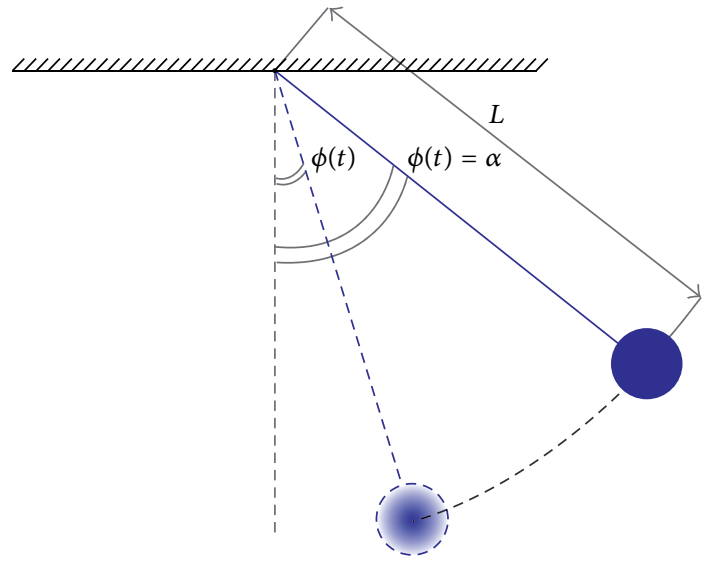

FIgURE 2: A nonlinear pendulum.

\section{Solution of Nonlinear Pendulum}

The pendulum [56-58] of Figure 2 can be modelled by (19). Nevertheless, we need to redefine the variables and parameters of (19), resulting in the following:

$$
\phi^{\prime \prime}+\lambda \sin (\phi)=0, \quad \phi(0)=\alpha, \quad \phi^{\prime}(0)=0,
$$

considering

$$
\lambda=\frac{g}{L},
$$

where prime denotes differentiation with respect to time $t, L$ is the length of the pendulum, $g$ is acceleration due to gravity, and $\alpha$ is the initial angle of displacement $\phi$.

Taking into account the above considerations and substituting $\eta=t$ into (29), results that the approximate solution (29) obtained by using NDHPM can be considered, also, as the solution $\phi(t)$ of the nonlinear pendulum problem (44).

7.1. Solution Obtained by Using HPM and Laplace-Padé Posttreatment. Although approximate solutions (35) and (41) possess good accuracy for cantilever problem, we need to increase the order of Taylor expansion for the sin term in (23) to obtain a highly accurate solution for pendulum problem, compared to other reported solutions [50-55]. Therefore, we propose to use a 22th-order Taylor series expansion of $\sin$ term, resulting in the following:

$$
\begin{aligned}
P(\phi)=\phi^{\prime \prime}+\lambda(\phi & -\frac{1}{6} \phi^{3}+\frac{1}{120} \phi^{5}-\frac{1}{5040} \phi^{7}+\frac{1}{362880} \phi^{9} \\
& -\frac{1}{39916800} \phi^{11}+\frac{1}{6227020800} \phi^{13} \\
& -\frac{1}{1307674368000} \phi^{15} \\
& +\frac{1}{355687428096000} \phi^{17} \\
& -\frac{1}{121645100408832000} \phi^{19} \\
& \left.+\frac{1}{51090942171709440000} \phi^{21}\right)=0
\end{aligned}
$$

where initial conditions are $\phi(0)=\alpha$ and $\phi^{\prime}(0)=0$.

A homotopy formulation that can generate a power series solution for this problem is

$$
(1-p)\left(L(v)-L\left(\phi_{0}\right)\right)+p(P(v))=0,
$$

where linear operator $L$ and trial function $\phi_{0}$ are taken from (31) and (32), respectively.

Substituting (5) into (47) and reordering and equating terms with the same $p$-powers, we obtain the following set of linear differential equations:

$$
\begin{gathered}
p^{0}: v_{0}^{\prime \prime}=0, \quad v_{0}(0)=\alpha, \quad v_{0}^{\prime}(0)=0 \\
p^{1}: v_{1}^{\prime \prime}+\lambda\left(v_{0}-\frac{1}{6} v_{0}^{3}+\frac{1}{120} v_{0}^{5}-\frac{1}{5040} v_{0}^{7}+\frac{1}{362880} v_{0}^{9}\right. \\
-\frac{1}{39916800} v_{0}^{11}+\frac{1}{6227020800} v_{0}^{13} \\
-\frac{1}{1307674368000} v_{0}^{15} \\
+\frac{1}{355687428096000} v_{0}^{17} \\
-\frac{1}{121645100408832000} v_{0}^{19} \\
\left.+\frac{1}{51090942171709440000} v_{0}^{21}\right)=0 \\
v_{1}(0)=0, \quad v_{1}^{\prime}(0)=0
\end{gathered}
$$


by solving (48), we obtain

$$
\begin{gathered}
v_{0}=\alpha \\
v_{1}=\left(-\frac{1}{2} \alpha+\frac{1}{12} \alpha^{3}-\frac{1}{240} \alpha^{5}+\frac{1}{10080} \alpha^{7}-\frac{1}{725760} \alpha^{9}\right. \\
+\frac{1}{79833600} \alpha^{11}-\frac{1}{12454041600} \alpha^{13} \\
+\frac{1}{2615348736000} \alpha^{15}-\frac{1}{711374856192000} \alpha^{17} \\
+\frac{1}{243290200817664000} \alpha^{19} \\
\left.-\frac{1}{102181884343418880000} \alpha^{21}\right) \lambda t^{2}
\end{gathered}
$$

where the subsequent iterations are calculated by using Maple CAS software.

Substituting solutions (49) into (5) and calculating the limit when $p \rightarrow 1$, we can obtain the 12th-order approximation:

$$
\phi(t)=\lim _{p \rightarrow 1}\left(\sum_{i=0}^{12} v_{i} p^{i}\right) .
$$

Expression (50) is too large to be written here; nevertheless, it can be simplified considering particular cases. For example, if we consider $\alpha=1^{\circ}$ and $\lambda=1$, we get the following results:

$$
\begin{aligned}
\phi(t)= & 0.0174532925200-0.00872620321865 t^{2} \\
& +0.000727072847969 t^{4}-2.42099213449 \times 10^{-5} t^{6} \\
& +4.28299598028 \times 10^{-7} t^{8}-4.35845271451 \\
& \times 10^{-9} t^{10}+5.75330823834 \times 10^{-12} t^{12} \\
& +1.31315973780 \times 10^{-12} t^{14}-5.55443572871 \\
& \times 10^{-14} t^{16}+1.62530684769 \times 10^{-15} t^{18} \\
& -3.65740303058 \times 10^{-17} t^{20}+6.06029996445 \\
& \times 10^{-19} t^{22}-5.08850528365 \times 10^{-21} t^{24}
\end{aligned}
$$

Now, (51) is transformed by the Laplace-Padé posttreatment. First, Laplace transformation is applied to (51) and then $1 / t$ is written in place of $s$. Then, Padé approximant [4/4] is applied and $1 / s$ is written in place of $t$. Finally, by using the inverse Laplace $s$ transformation, we obtain the modified approximate LPHPM solution:

$$
\begin{aligned}
\phi(t)= & -2.76933314632 \times 10^{-8} \cos (2.99987437966 t) \\
& +0.0174533202134 \cos (0.999980961654 t) .
\end{aligned}
$$

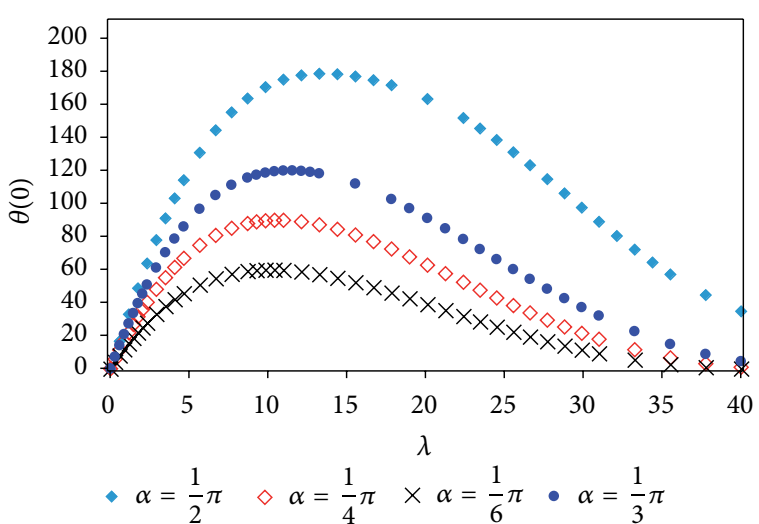

FIGURE 3: Terminal slope versus follower force for various angles of inclination $\alpha$.

Equation (52) is more compact and computationally efficient than power series solution (51). Thus, (52) can be used when accurate and handy expressions are needed. This process can be repeated for different values of $\alpha$ and $\lambda$.

We can express pendulum period in terms of

$$
T=4 \sqrt{\frac{1}{\lambda}} K(\epsilon), \quad \epsilon=\sin \left(\frac{\alpha}{2}\right),
$$

where $K$ is the complete elliptic function of the first kind formulated as

$$
K(\epsilon)=\int_{0}^{\pi / 2} \frac{1}{\sqrt{1-\epsilon^{2} \sin ^{2} \theta}} d \theta, \quad 0 \leq \epsilon<1 .
$$

For comparison purposes, we will normalize the value of $T$ to

$$
\widehat{T}=\frac{\sqrt{\lambda}}{4} T=K(\epsilon)
$$

Finally, $\widehat{T}$ is calculated for some values of $\alpha$ in Tables 3 and 4.

\section{Numerical Simulation and Discussion}

8.1. Cantilever Problem. Structures subjected to follower forces have been reported by several researchers $[50,55]$. In this work, we propose the solution for large deflections of a cantilever beam subjected to a follower force reported in [55]. Therefore, by using approximate solution (29) and (21), we show in Figure 3 the relationship between terminal slope $\theta(0)$ and load parameter $\lambda$ for some values of deflection angle in the range $\alpha \in\left(0^{\circ}, 180^{\circ}\right)$. Flutter instability occurs, and the maximum value of terminal slope is approximately $\theta(0)=2 \alpha$ as reported in $[50,55]$.

On one side, considering a fixed value for $\alpha=\pi / 4$, we show in Figure 4 the deformed curves of a beam under different values of load parameter $\lambda$. First, the cantilever beam is a straight line, and it is deformed gradually as the amplitude of the end-concentrated follower force increases, until the curvature of the beam is notorious for $\lambda=24$. Additionally, 
TABLE 2: Terminal slope $\theta(0)$ and coordinates $[\xi(0), \zeta(0)]$ of cantilever beam under normal follower forces $(\alpha=\pi / 2)$.

\begin{tabular}{lccccccccc}
\hline Method & D.M. [55] & NDHPM (29) & HPM [50] & D.M. [55] & NDHPM (29) & HPM [50] & D.M. [55] & NDHPM (29) & HPM [50] \\
$\lambda$ & $\theta(0)^{\circ}$ & $\theta(0)^{\circ}$ & $\theta(0)^{\circ}$ & $\xi(0)$ & $\xi(0)$ & $\xi(0)$ & $\zeta(0)$ & $\zeta(0)$ & $\zeta(0)$ \\
\hline 2.00 & 55.48 & 55.44 & 55.48 & 0.7674 & 0.7677 & 0.7673 & 0.5738 & 0.5735 \\
4.00 & 101.78 & 101.84 & 101.84 & 0.3428 & 0.3419 & 0.3420 & 0.7862 & 0.7866 \\
8.00 & 157.94 & 158.20 & 158.06 & -0.0739 & -0.0765 & -0.0749 & 0.6336 & 0.6323 & 0.5739 \\
13.75 & 180.00 & 178.75 & 179.81 & 0.0000 & 0.0104 & 0.0019 & 0.4570 & 0.4577 & 0.4564 \\
16.00 & 177.56 & 176.27 & 177.22 & 0.0910 & 0.1009 & 0.0938 & 0.4212 & 0.4215 \\
24.00 & 140.04 & 141.94 & 141.16 & 0.4348 & 0.4310 & 0.4320 & 0.1588 & 0.1732 & 0.4206 \\
36.00 & 55.64 & 54.20 & 54.51 & 0.2855 & 0.2726 & 0.2740 & -0.4546 & -0.4619 & -0.4608 \\
\hline
\end{tabular}

TABLE 3: Numerical comparison for $\widehat{T}$ of nonlinear pendulum. Considering $\lambda=1$.

\begin{tabular}{lcccc}
\hline$\alpha^{\circ}$ & $\widehat{T}(55)$ & HPM (50) & LPHPM & NDHPM (29) \\
\hline 1.00000000 & 1.5708262330620782 & 1.5708262330620789 & 1.5708262328912635 & 1.5708262330620782 \\
10.00000000 & 1.5737921309247680 & 1.5737921309137186 & 1.5737904171285648 & 1.5737921309298985 \\
50.00000000 & 1.6489952184785310 & 1.6489951818036827 & 1.6478388934763048 & 1.6489966814551095 \\
100.00000000 & 1.9355810960047220 & 1.9356331885927838 & 1.9133053425965654 & 1.9345771658441948 \\
120.00000000 & 2.1565156474996432 & 2.1562170331637955 & 2.1071446302159819 & 2.1435695247049310 \\
130.00000000 & 2.3087867981671961 & 2.3080949709723178 & 2.2390737258149828 & 2.2708015298391809 \\
140.00000000 & 2.5045500790016340 & 2.5047278166695662 & 2.4091002654586270 & 2.4052794658306813 \\
150.00000000 & 2.7680631453687676 & 2.7723737183655317 & 2.6410091037967786 & 2.5354467979775911 \\
160.00000000 & 3.1533852518878388 & 3.1570008973230000 & 2.9887412725547848 & - \\
170.00000000 & 3.8317419997841462 & 3.8074862454595039 & 3.6247866486947020 & - \\
175.00000000 & 4.5202232749476581 & 4.5078737329373597 & 4.2924790748606028 & - \\
177.00000000 & 5.0298609371127860 & 5.0733536048198848 & 4.79490944850198683 & - \\
179.00000000 & 6.127778824526868 & 4.631673755961933 & 5.88740406465829867 & - \\
179.90000000 & 8.430255141254121 & - & 8.18871358260360318 & \\
179.99999000 & 17.64055690983073 & - & 17.3989702100090882 & - \\
179.99999999 & 24.548351581120853 & - & 24.2491870075566718 & - \\
\hline
\end{tabular}

when the load parameter is larger than the flutter load, the beam deforms towards the opposite direction as reported in [50]. On the other side, we consider a fixed value for load parameter $\lambda=16$ and different values of $\alpha$ as shown in Figure 5, resulting that the deflection and terminal slope $\theta(0)$ are influenced by the angle $\alpha$ of follower force producing a notorious bending as $\alpha$ increases reaching the extreme case at $\alpha=\pi / 2$.

Considering a normal angle $\alpha=\pi / 2$, we show in Table 1 the terminal slope $\theta(0)$ (using (21)) versus different values of load parameter $\lambda$. In such table, we compare the proposed solutions (29), (35), (41), LPHPM/LPNDHPM approximations (see (37), and (43)) to exact solution [51], a shooting method solution [53], and HPM solution reported in [50]. Our proposed approximate solutions are in good agreement with the other reported approximations. In particular, (29) and LPNDHPM approximations (see (43)) exhibited the best accuracy of all proposed solutions of this work, with a similar accuracy compared to the results reported in [50]. Furthermore, we showed in Table 2, the terminal slope $\theta(0)$ and coordinates $[\xi(0), \zeta(0)]$ (see $(22)$ ) of cantilever beam under normal follower forces $(\alpha=\pi / 2)$, resulting that our proposed solution (29) exhibited an accuracy in good agreement with the values obtained by a direct method
(D.M.) [55] and the results reported in [50]. For this work, the cantilever beam is considered elastic and ideal; then, Figures 4 and 5 present an ideally deformed beam. Further work should be performed in order to include nonideal effects due to material characteristics of cantilever.

From above, we can conclude that the deflection and terminal slope are governed by the deflection angle and magnitude of the follower force. This means that the bending of the beam becomes more notorious as the deflection angle or the magnitude of the follower force increases [50].

8.2. Pendulum Problem. On one hand, in $[56,58]$ the authors achieve approximate solutions for pendulum period, with relative error $<2 \%$ for $\alpha \leq 130^{\circ}$, and $<1 \%$ for $\alpha \leq 150^{\circ}$, respectively. On the other hand, in Table 3 and Table 4 we show a comparison between $\widehat{T}, T / 4$ calculated using (29) and (50) and approximations calculated by LPHPM procedure explained in Section 7.1 (considering $\lambda=1$ ). The value $\widehat{T}$ is obtained, applying Newton-Raphson method to equation $\phi(t)=0$, that is, calculating the first crossing of $\phi(t)$ by zero since the initial angle $\phi(0)$. In Table 4 , we show the relative error (RE) for the range of $\alpha \in\left(0^{\circ}, 180^{\circ}\right)$, resulting that our proposed solutions are in good agreement with exact 
TABLE 4: Relative error (RE) comparison for $\widehat{T}$ of nonlinear pendulum (see Table 3 ).

\begin{tabular}{lcccc}
\hline$\alpha^{\circ}$ & $\widehat{T}(55)$ & R.E. HPM (50) & R.E. LPHPM & R.E. NDHPM (29) \\
\hline 1.00000000 & 1.5708262330620782 & $-4.355 e-16$ & $1.087 e-10$ & $-3.247 e-20$ \\
10.00000000 & 1.5737921309247680 & $7.021 e-12$ & $1.089 e-06$ & $-3.260 e-12$ \\
50.00000000 & 1.6489952184785310 & $2.224 e-08$ & 0.0007012 & $-8.872 e-07$ \\
100.00000000 & 1.9355810960047220 & $-2.691 e-05$ & 0.01151 & 0.0005187 \\
120.00000000 & 2.1565156474996432 & 0.0001385 & 0.02289 & 0.006003 \\
130.00000000 & 2.3087867981671961 & 0.0002996 & 0.03019 & 0.01645 \\
140.00000000 & 2.5045500790016340 & $-7.097 e-05$ & 0.03811 & 0.03964 \\
150.00000000 & 2.7680631453687676 & -0.001557 & 0.0459 & 0.08404 \\
160.00000000 & 3.1533852518878388 & -0.001147 & 0.05221 & - \\
170.00000000 & 3.8317419997841462 & 0.00633 & 0.05401 & - \\
175.00000000 & 4.5202232749476581 & 0.002732 & 0.05038 & - \\
177.00000000 & 5.0298609371127860 & $-0.86468 e-2$ & $0.4671132 e-1$ & - \\
179.00000000 & 6.127778824526868 & 0.244151 & $0.3922706 e-1$ & - \\
179.90000000 & 8.430255141254121 & - & $0.28651749 e-1$ & - \\
179.99999000 & 17.64055690983073 & - & $0.1369703 e-1$ & - \\
179.99999999 & 24.548351581120853 & - & 0.01222747 & - \\
\hline
\end{tabular}

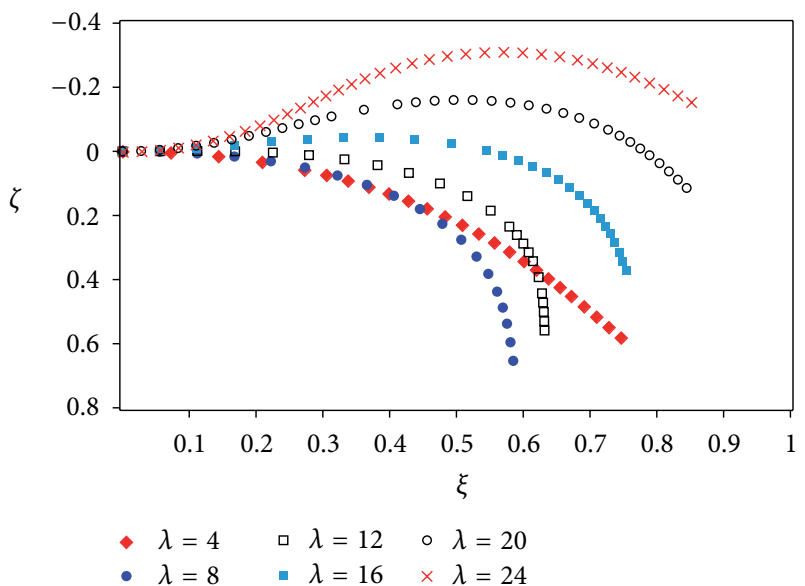

FIGURE 4: Deflection curves of a cantilever beam axis under several follower forces, considering $\alpha=\pi / 4$.

results. Furthermore, LPHPM solution can predict the $\widehat{T}$ for $\alpha=179.99999999^{\circ}$ exhibiting a RE of 0.01222747 , which is highly accurate compared to the results obtained in $[56,58]$.

The coupling of Laplace-Padé and HPM method was a key factor to increase the accuracy range until $\phi(0)$ value is really close to $180^{\circ}$. Laplace-Padé posttreatment was able to deal with the truncate power series, in order to obtain better accuracy for angles close to $\alpha=180^{\circ}$ although the behaviour was different for small values of $\alpha$. However, in general terms, the Laplace-Padé posttreatment generates handy and computationally more efficient expressions (see (51) versus (52)). Furthermore, we can appreciate from Tables 3 and 4 that HPM and NDHPM methods do not present useful $\widehat{T}$ values for $\alpha>150^{\circ}$ and $\alpha>179^{\circ}$, due to numerical noise of the proposed approximations. Fortunately, LPHPM approximations achieve a good accuracy within the range $1^{\circ} \leq \alpha \leq 179.99999999^{\circ}$. From the above discussion

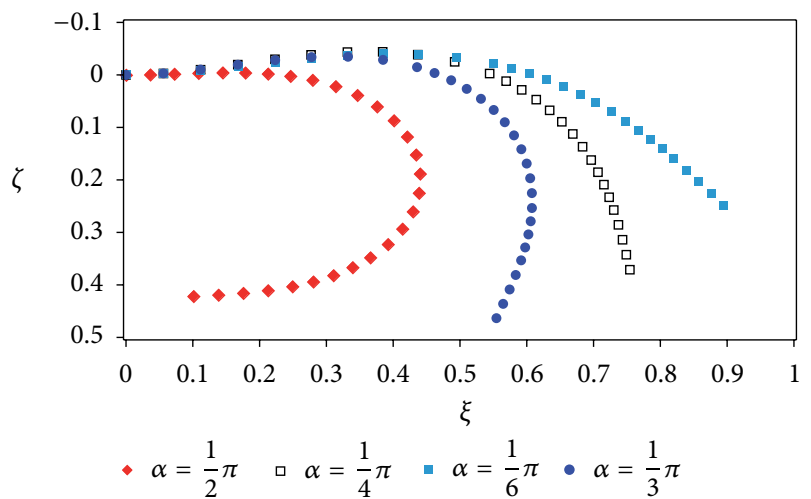

FIGURE 5: Deflection curves beam axis under follower force $\lambda=16$ for various angles of inclination $\alpha$.

we can conclude that, as the initial angle of displacement $\alpha$ approximates to the vicinity $180^{\circ}$, the pendulum period increases notoriously.

Finally, further research should be done in order to obtain approximate solutions of the pendulum problem involving additional nonlinear effects like friction and mass, among others.

\section{Conclusions}

In this paper we presented the solution for two nonlinear problems related by the same differential equation. The first one was the large deflection of a cantilever beam under a terminal follower force. The second one was the nonlinear pendulum problem. We proposed a series of solutions obtained by NDHPM, HPM, and combinations with LaplacePadé posttreatment. For both problems, results exhibited high accuracy, which are in good agreement with the results reported by other works. In particular, for the pendulum 
case, we succeeded to predict, accurately, the period for an initial angle up to $179.99999999^{\circ}$. By means of two case studies, we confirmed that NDHPM, HPM, LPNDHPM, and LPHPM are powerful and useful methods; all of them capable of generating highly accurate approximations for nonlinear problems.

\section{Acknowledgments}

The authors gratefully acknowledge the financial support provided by the National Council for Science and Technology of Mexico (CONACyT) through Grant CB-2010-01 no. 157024. The authors would like to express their gratitude to RogelioAlejandro Callejas-Molina and Roberto Ruiz-Gomez for their contribution to this paper.

\section{References}

[1] J.-H. He, "Asymptotic methods for solitary solutions and compactons," Abstract and Applied Analysis, vol. 2012, Article ID 916793, 130 pages, 2012.

[2] J.-H. He, "Comparison of homotopy perturbation method and homotopy analysis method," Applied Mathematics and Computation, vol. 156, no. 2, pp. 527-539, 2004.

[3] J.-H. He, "An elementary introduction to the homotopy perturbation method," Computers \& Mathematics with Applications, vol. 57, no. 3, pp. 410-412, 2009.

[4] J.-H. He, "Homotopy perturbation technique," Computer Methods in Applied Mechanics and Engineering, vol. 178, no. 3-4, pp. 257-262, 1999.

[5] J.-H. He, "The homotopy perturbation method nonlinear oscillators with discontinuities," Applied Mathematics and Computation, vol. 151, no. 1, pp. 287-292, 2004.

[6] J.-H. He, "Homotopy perturbation method: a new nonlinear analytical technique," Applied Mathematics and Computation, vol. 135, no. 1, pp. 73-79, 2003.

[7] J.-H. He, "A coupling method of a homotopy technique and a perturbation technique for non-linear problems," International Journal of Non-Linear Mechanics, vol. 35, no. 1, pp. 37-43, 2000.

[8] J. H. He, "Application of homotopy perturbation method to nonlinear wave equations," Chaos, Solitons and Fractals, vol. 26, no. 3, pp. 695-700, 2005.

[9] J.-H. He, "Homotopy perturbation method for solving boundary value problems," Physics Letters A, vol. 350, no. 1-2, pp. 8788, 2006.

[10] D. D. Ganji, "A semi-Analytical technique for non-linear settling particle equation of Motion," Journal of Hydro-Environment Research, vol. 6, no. 4, pp. 323-327, 2012.

[11] M. Sheikholeslami and D. D. Ganji, "Heat transfer of Cu-water nanofluid flow between parallel plates," Powder Technology, vol. 235, pp. 873-879, 2013.

[12] S. S. Ganji, D. D. Ganji, M. G. Sfahani, and S. Karimpour, "Application of AFF and HPM to the systems of strongly nonlinear oscillation," Current Applied Physics, vol. 10, no. 5, pp. 1317-1325, 2010.

[13] D. D. Ganji, H. B. Rokni, M. G. Sfahani, and S. S. Ganji, "Approximate traveling wave solutions for coupled WhithamBroer-Kaup shallow water," Advances in Engineering Software, vol. 41, no. 7-8, pp. 956-961, 2010.
[14] A. Barari, M. Omidvar, A. R. Ghotbi, and D. D. Ganji, "Application of homotopy perturbation method and variational iteration method to nonlinear oscillator differential equations," Acta Applicandae Mathematicae, vol. 104, no. 2, pp. 161-171, 2008.

[15] H. Koçak and A. Yildirim, "Numerical solution of 3D Green's function for the dynamic system of anisotropic elasticity," Physics Letters A, vol. 373, no. 35, pp. 3145-3150, 2009.

[16] Y. Khan, H. Vazquez-Leal, and Q. Wu, "An efficient iterated method for mathematical biology model," Neural Computing and Applications, pp. 1-6, 2012.

[17] Y. Khan, Q. Wu, N. Faraz, A. Yildirim, and M. Madani, "A new fractional analytical approach via a modified RiemannLiouville derivative," Applied Mathematics Letters, vol. 25, no. 10, pp. 1340-1346, 2012.

[18] N. Faraz and Y. Khan, "Analytical solution of electrically conducted rotating flow of a second grade fluid over a shrinking surface," Ain Shams Engineering Journal, vol. 2, no. 34, pp. 221226, 2011

[19] Y. Khan, Q. Wu, N. Faraz, and A. Yildirim, "The effects of variable viscosity and thermal conductivity on a thin film flow over a shrinking/stretching sheet," Computers \& Mathematics with Applications, vol. 61, no. 11, pp. 3391-3399, 2011.

[20] J. Biazar and H. Aminikhah, "Study of convergence of homotopy perturbation method for systems of partial differential equations," Computers \& Mathematics with Applications, vol. 58, no. 11-12, pp. 2221-2230, 2009.

[21] J. Biazar and H. Ghazvini, "Convergence of the homotopy perturbation method for partial differential equations," Nonlinear Analysis, vol. 10, no. 5, pp. 2633-2640, 2009.

[22] J. Biazar, F. Badpeima, and F. Azimi, "Application of the homotopy perturbation method to Zakharov-Kuznetsov equations," Computers \& Mathematics with Applications, vol. 58, no. 11-12, pp. 2391-2394, 2009.

[23] J. Biazar and H. Ghazvini, "Homotopy perturbation method for solving hyperbolic partial differential equations," Computers \& Mathematics with Applications, vol. 56, no. 2, pp. 453-458, 2008.

[24] J. Biazar and H. Ghazvini, "Exact solutions for non-linear Schrödinger equations by He's homotopy perturbation method," Physics Letters A, vol. 366, no. 1-2, pp. 79-84, 2007.

[25] M. Fathizadeh, M. Madani, Y. Khan, N. Faraz, A. Yildirim, and S. Tutkun, "An effective modification of the homotopy perturbation method for mhd viscous flow over a stretching sheet," Journal of King Saud University-Science, 2011.

[26] M. Madani, M. Fathizadeh, Y. Khan, and A. Yildirim, "On the coupling of the homotopy perturbation method and Laplace transformation," Mathematical and Computer Modelling, vol. 53, no. 9-10, pp. 1937-1945, 2011.

[27] H. Vázquez-Leal, U. Filobello-Niño, R. Castañeda-Sheissa, A. Sarmiento-Reyes, and J. Sanchez Orea, "High accurate simple approximation of normal distribution integral," Mathematical Problems in Engineering, vol. 2012, Article ID 124029, 22 pages, 2012.

[28] H. Vázquez-Leal, U. Filobello-Niño, R. Castañeda-Sheissa, L. Hernández-Martínez, and A. Sarmiento-Reyes, "Modified HPMs inspired by homotopy continuation methods," Mathematical Problems in Engineering, vol. 2012, Article ID 309123, 19 pages, 2012.

[29] U. Filobello-Niño, H. Vázquez-Leal, R. Castañeda-Sheissa et al., "An approximate solution of blasius equation by using hpm method," Asian Journal of Mathematics and Statistics, vol. 5, no. 2, pp. 50-59, 2012. 
[30] D. Ağırseven and T. Öziş, "An analytical study for Fisher type equations by using homotopy perturbation method," Computers \& Mathematics with Applications, vol. 60, no. 3, pp. 602-609, 2010.

[31] T. Öziş and D. Ağırseven, "He’s homotopy perturbation method for solving heat-like and wave-like equations with variable coefficients," Physics Letters A, vol. 372, no. 38, pp. 5944-5950, 2008.

[32] T. Öziş and A. Yildirim, "A note on He’s homotopy perturbation method for van der Pol oscillator with very strong nonlinearity," Chaos, Solitons and Fractals, vol. 34, no. 3, pp. 989-991, 2007.

[33] D. D. Ganji and A. Rajabi, "Assessment of homotopyperturbation and perturbation methods in heat radiation equations," International Communications in Heat and Mass Transfer, vol. 33, no. 3, pp. 391-400, 2006.

[34] M. Rafei, D. D. Ganji, and H. Daniali, "Solution of the epidemic model by homotopy perturbation method," Applied Mathematics and Computation, vol. 187, no. 2, pp. 1056-1062, 2007.

[35] D. D. Ganji, "The application of He's homotopy perturbation method to nonlinear equations arising in heat transfer," Physics Letters A, vol. 355, no. 4-5, pp. 337-341, 2006.

[36] D. D. Ganji and A. Sadighi, "Application of homotopyperturbation and variational iteration methods to nonlinear heat transfer and porous media equations," Journal of Computational and Applied Mathematics, vol. 207, no. 1, pp. 24-34, 2007.

[37] M. Sheikholeslami, H. R. Ashorynejad, D. D. Ganji, and A. Yildirim., "Homotopy perturbation method for threedimensional problem of condensation film on inclined rotating disk," Scientia Iranica, vol. 19, no. 3, pp. 437-442, 2012.

[38] D. D. Ganji, H. Tari, and M. B. Jooybari, "Variational iteration method and homotopy perturbation method for nonlinear evolution equations," Computers \& Mathematics with Applications, vol. 54, no. 7-8, pp. 1018-1027, 2007.

[39] J.-H. He, "Homotopy perturbation method with an auxiliary term," Abstract and Applied Analysis, vol. 2012, Article ID 857612, 7 pages, 2012.

[40] Y. Yamamoto, C. Dang, Y. Hao, and Y. C. Jiao, "An aftertreatment technique for improving the accuracy of Adomian's decomposition method," Computers \& Mathematics with Applications, vol. 43, no. 6-7, pp. 783-798, 2002.

[41] N. H. Sweilam and M. M. Khader, "Exact solutions of some coupled nonlinear partial differential equations using the homotopy perturbation method," Computers \& Mathematics with Applications, vol. 58, no. 11-12, pp. 2134-2141, 2009.

[42] S. Momani, G. H. Erjaee, and M. H. Alnasr, "The modified homotopy perturbation method for solving strongly nonlinear oscillators," Computers \& Mathematics with Applications, vol. 58, no. 11-12, pp. 2209-2220, 2009.

[43] Y. Khan and N. Faraz, "Application of modified Laplace decomposition method for solving boundary layer equation," Journal of King Saud University -Science, vol. 23, no. 1, pp. 115-119, 2011.

[44] D. Bahuguna, A. Ujlayan, and D. N. Pandey, "A comparative study of numerical methods for solving an integro-differential equation," Computers \& Mathematics with Applications, vol. 57, no. 9, pp. 1485-1493, 2009.

[45] S. Momani and V. S. Ertürk, "Solutions of non-linear oscillators by the modified differential transform method," Computers \& Mathematics with Applications, vol. 55, no. 4, pp. 833-842, 2008.

[46] A. Gökdoğan, M. Merdan, and A. Yildirim, "The modified algorithm for the differential transform method to solution of Genesio systems," Communications in Nonlinear Science and Numerical Simulation, vol. 17, no. 1, pp. 45-51, 2012.
[47] P.-Y. Tsai and C.-K. Chen, "An approximate analytic solution of the nonlinear Riccati differential equation," Journal of the Franklin Institute, vol. 347, no. 10, pp. 1850-1862, 2010.

[48] A. E. Ebaid, "A reliable aftertreatment for improving the differential transformation method and its application to nonlinear oscillators with fractional nonlinearities," Communications in Nonlinear Science and Numerical Simulation, vol. 16, no. 1, pp. 528-536, 2011.

[49] M. Merdan, A. Gökdoğan, and A. Yildirim, "On the numerical solution of the model for HIV infection of $C D 4^{+} \mathrm{T}$ cells," Computers \& Mathematics with Applications, vol. 62, no. 1, pp. 118-123, 2011.

[50] Y.-G. Wang, W.-H. Lin, and N. Liu, "A homotopy perturbationbased method for large deflection of a cantilever beam under a terminal follower force," International Journal for Computational Methods in Engineering Science and Mechanics, vol. 13, no. 3, pp. 197-201, 2012.

[51] B. N. Rao and G. V. Rao, "On the large deflections of cantilever beams with end rotational load," Zeitschrift Fur Angewandte Mathematik Und Mechanik, vol. 6, pp. 507-508, 1986.

[52] B. N. Rao and G. V. Rao, "Large deflections of a nonuniform cantilever beam with end rotational load," Forschung im Ingenieurwesen, vol. 54, no. 1, pp. 24-26, 1988.

[53] B. N. Rao and G. V. Rao, "Large deflections of a cantilever beam subjected to a rotational distributed loading," Forschung im Ingenieurwesen, vol. 55, no. 4, pp. 116-120, 1989.

[54] J. H. Argyris and S Symeonidis, "Nonlinear finite element analysis of elastic systems under nonconservative loading: natural formulation. I. Quasistatic problems," Computer Methods in Applied Mechanics and Engineering, vol. 26, no. 1, pp. 75-123, 1981.

[55] B. S. Shvartsman, "Large deflections of a cantilever beam subjected to a follower force," Journal of Sound and Vibration, vol. 304, no. 3-5, pp. 969-973, 2007.

[56] A. Beléndez, A. Hernández, T. Beléndez, C. Neipp, and A. Márquez, "Application of the homotopy perturbation method to the nonlinear pendulum," European Journal of Physics, vol. 28, no. 1, article 010, pp. 93-104, 2007.

[57] A. Beléndez, E. Arribas, M. Ortuño, S. Gallego, A. Márquez, and I. Pascual, "Approximate solutions for the nonlinear pendulum equation using a rational harmonic representation," Computers \& Mathematics with Applications, vol. 64, no. 6, pp. 1602-1611, 2012.

[58] A. Beléndez, C. Pascual, M. L. Lvarez, D. I. Méndez, M. S. Yebra, and A. Hernndez, "Higher order analytical approximate solutions to the nonlinear pendulum by He's homotopy method," Physica Scripta, vol. 79, no. 1, Article ID 015009, 2009.

[59] P. Brzeski, P. Perlikowski, S. Yanchuk, and T. Kapitaniak, "The dynamics of the pendulum suspended on the forced Duffing oscillator," Journal of Sound and Vibration, vol. 331, no. 24, pp. 5347-5357, 2012.

[60] G. A. Baker, Essentials of Padé Approximants, Academic Express, London, UK, 1975. 


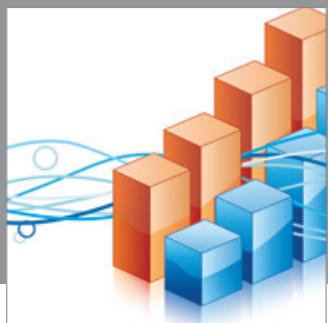

Advances in

Operations Research

mansans

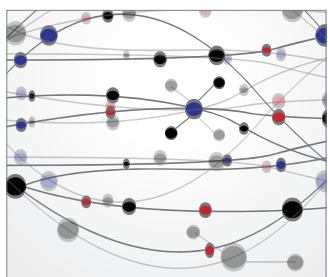

The Scientific World Journal
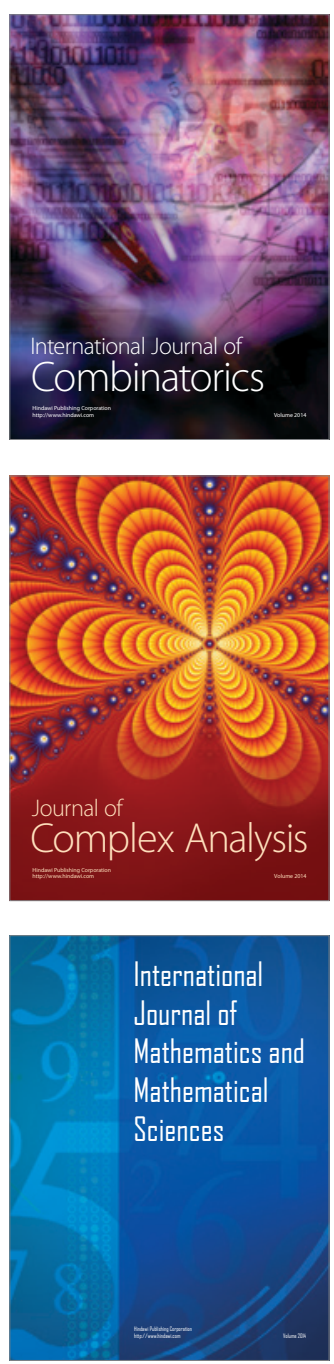
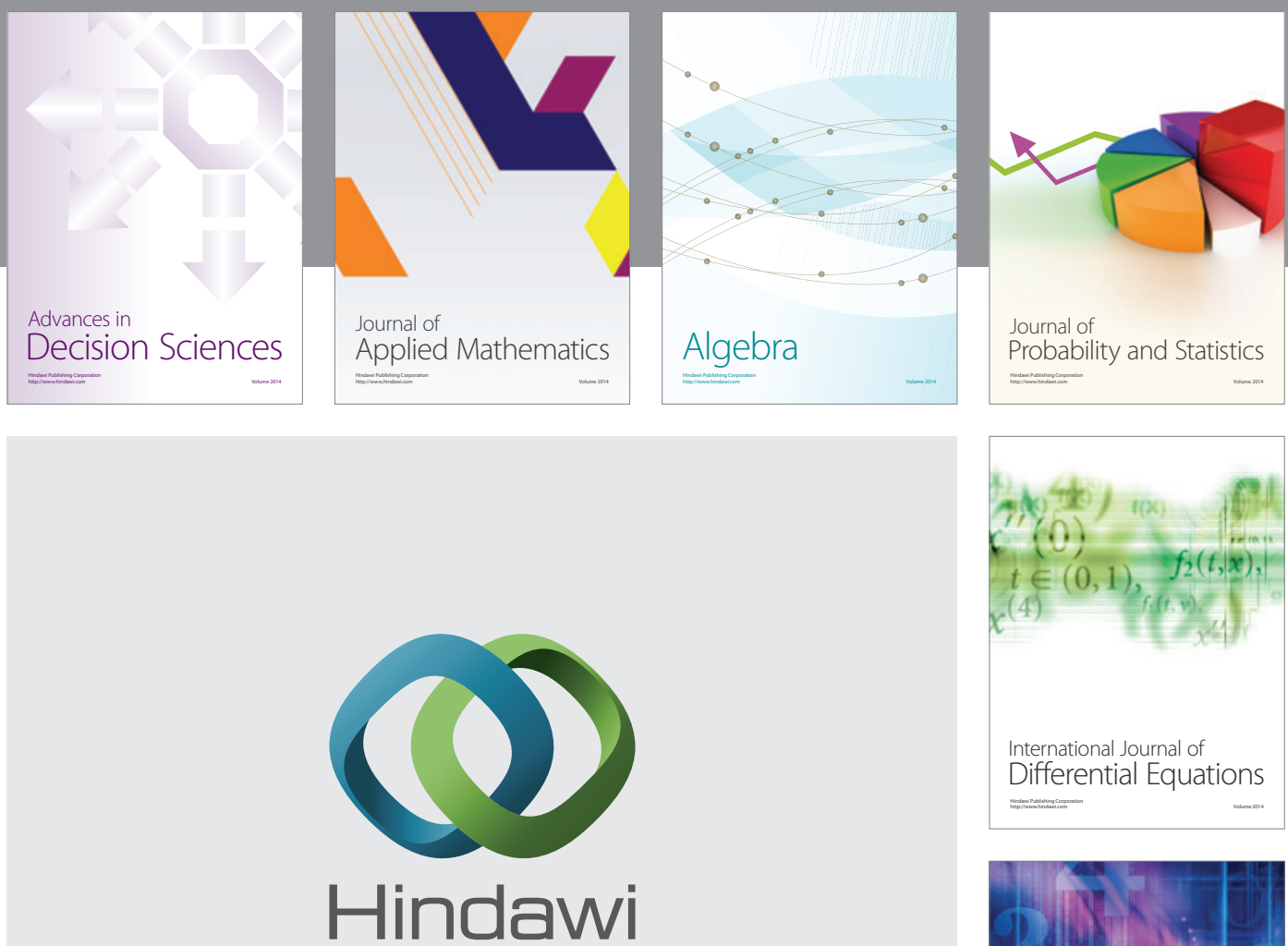

Submit your manuscripts at http://www.hindawi.com
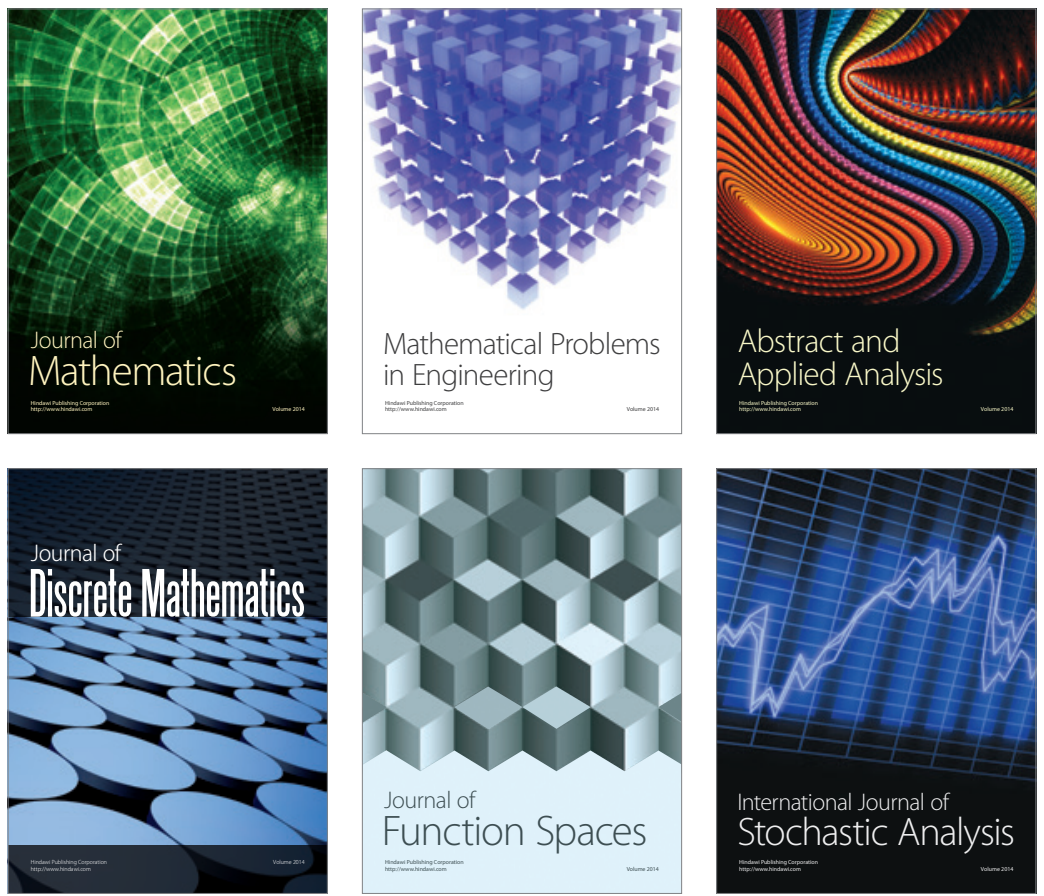

Journal of

Function Spaces

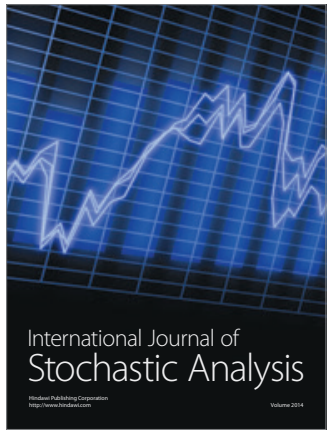

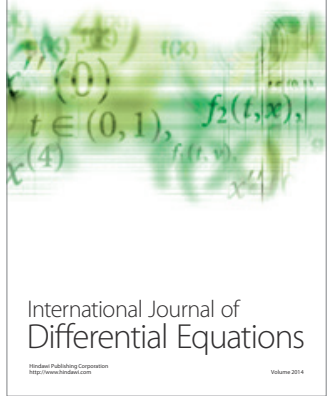
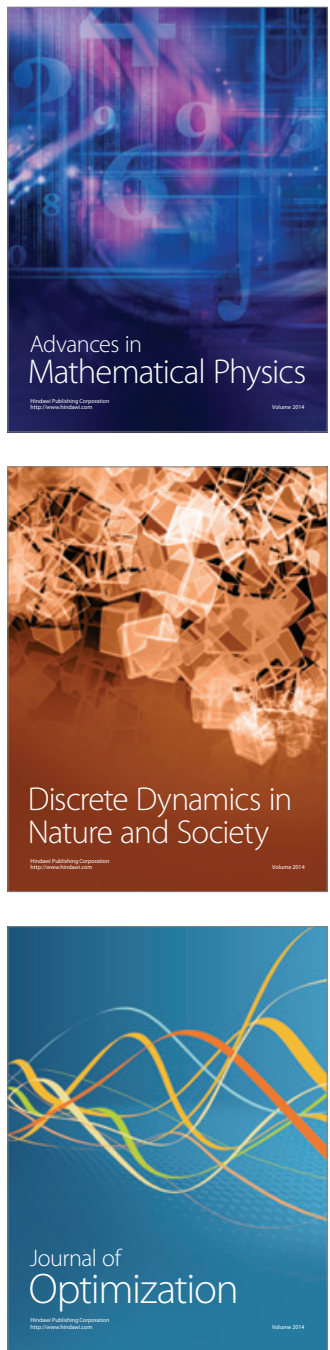